\title{
A new intermediate intercalate in superconducting sodium-doped hafnium nitride chloride
}

\author{
Judith Oró-Solé, ${ }^{a}$ Carlos Frontera, ${ }^{a}$ Benjamín Martínez, ${ }^{a}$ Daniel Beltrán-Porter, ${ }^{b}$ Maria Rosa Palacín ${ }^{a}$ and \\ Amparo Fuertes*a
}

Received (in Cambridge, UK) 24th March 2005, Accepted 25th April 2005

First published as an Advance Article on the web 24th May 2005

DOI: $10.1039 / \mathrm{b504289e}$

\begin{abstract}
A new phase has been observed during the sodium intercalation of hafnium nitride chloride as intermediate between the host $\beta$-HfNCl and the already reported $\mathrm{Na}_{0.29} \mathrm{HfNCl}$ with $T_{\mathrm{c}}$ of $24 \mathrm{~K}$; the new intermediate shows interlayer spacings ranging from 9.48 to $9.67 \AA$, corresponds to a second stage intercalate of $\mathrm{HfNCl}$ and is superconducting with a critical temperature of $20 \mathrm{~K}$.
\end{abstract}

Intercalated zirconium and hafnium nitride halides have been reported to exhibit superconductivity with high critical temperatures within non oxidic compounds. ${ }^{1,2}$ The pristine compounds MNX ( $\mathrm{M}=\mathrm{Zr}$, Hf; $\mathrm{X}=\mathrm{Cl}, \mathrm{Br}$, I) show two layered polytypes, $\alpha$ and $\beta$ phases, isotypic to $\mathrm{FeOCl}$ and SmSI respectively. ${ }^{3,46}$ The structure of the $\beta$ phase can be described as a stacking along the $\mathrm{c}$ axis of double layers with composition [X-M-N-N-M-X], separated by a van der Waals gap. Superconductivity in $\mathrm{ZrNX}$ or HfNX is induced by intercalation of alkaline metals or Lewis bases as cobaltocene or pyridine into the van der Waals gap. ${ }^{1-3,5,7}$ For sodium intercalated hafnium nitride chloride a superconducting phase has been reported with critical temperatures of 20, 23 or $24 \mathrm{~K}^{8-11}$ and in lithium intercalated $\mathrm{HfNCl}$, critical temperatures of $18,20,24$ and $26 \mathrm{~K}$ have been observed in different samples showing in some cases co-intercalated organic molecules. ${ }^{2,12,13}$ In both systems as well as in doped zirconium nitride chloride that shows lower critical temperatures (close to $12 \mathrm{~K}$ ), the influence on the critical temperature of factors such as the nature of the transition metal, the co-intercalated molecules and the doping level have still not been completely understood. The crystal structure of $\mathrm{Na}_{x} \mathrm{HfNCl}$ with $x=0.29$ has been reported as isotypic to YOF with space group $R \overline{3} m$ and crystal parameters $a=3.5892(3) \AA$ and $c=29.722(3) \AA .{ }^{8}$ The interlayer spacing is $1 / 3$ of the c parameter, being $9.80 \AA$ for $\mathrm{Na}_{0.29} \mathrm{HfNCl}$ and $9.22 \AA$ for the pristine. ${ }^{3}$

Staging during intercalation of layered compounds is a phenomenon that has been frequently observed in graphite and in transition metal dichalcogenides, in which a unit consisting of a guest layer followed by $n$ host layers (for a stage- $n$ compound) is repeated along the c axis. ${ }^{14,15}$ The phase $\mathrm{Na}_{0.29} \mathrm{HfNCl}$ has been described as a diluted stage 1 intercalate, with all the van der Waals gaps partially filled with sodium atoms. Here we report a new intercalated phase, with interlayer spacing between 9.48 and $9.67 \AA$ that is obtained as an intermediate in the intercalation process of the host to give $\mathrm{Na}_{0.29} \mathrm{HfNCl}$. The new phase shows a critical temperature of $20 \mathrm{~K}$ and can be interpreted as a second stage intercalate of $\mathrm{HfNCl}$.

*amparo.fuertes@icmab.es
$\mathrm{HfNCl}$ or $\mathrm{ZrNCl}$ were prepared by reaction of hafnium (Aldrich 99.5\%) or zirconium (Alfa 99.9\%) and ammonium chloride (Aldrich 99.99\%) in the stoichiometric ratio 1:1.1 at temperatures between 740 and $780{ }^{\circ} \mathrm{C}$ during 12 hours in sealed evacuated silica tubes. In a second step a temperature gradient of $100{ }^{\circ} \mathrm{C}$ was applied to the same reaction tube and $\mathrm{HfNCl}$ and $\mathrm{ZrNCl}$ were recrystallized by chemical vapour transport. Sodium intercalation reactions were performed by the treatment of $\mathrm{HfNCl}$ with Naphtyl-sodium solutions in tetrahydrofuran with concentrations between $0.025 \mathrm{M}$ and $1 \mathrm{M}$. The intercalation reaction time was 24 hours and the molar $\mathrm{Na}: \mathrm{HfNCl}$ ratios ranged between $0.5: 1$ and 50:1. Filling of the reaction tubes, intercalation reactions and handling of the products for subsequent characterization were performed in an argon filled glove box. Attempts to determine the sodium content by chemical analysis were hindered by the fact that some sodium de-intercalation was found to take place during washing of the samples. With comparative purposes, electrochemical lithium intercalation experiments were conducted both in $\mathrm{HfNCl}$ and $\mathrm{ZrNCl}$ in lithium cells as previously described. The cells were tested using a MacPile potentiostat (Biologic Science Instruments, Claix, France) in galvanostatic mode at a $\mathrm{C} / 200$ rate (i.e. intercalation of $1 \mathrm{~mol}$ of lithium ions per mol of compound). Magnetic susceptibility measurements were performed in a Quantum Design SQUID magnetometer down to $4 \mathrm{~K}$ in zero field cooled and field cooled conditions $(\mathrm{H}=30 \mathrm{G})$. Powder X ray diffraction patterns were taken on an INEL curved position sensitive CPS120 powder diffractometer using a rotating glass capillary $0.1 \mathrm{~mm}$ in diameter as sample holder. The angular range was $114^{\circ}$ and the radiation was $\mathrm{Cu} \mathrm{k} \alpha_{1}(\lambda=1.540598 \AA$ ) , obtained with a Ge (111) monochromator. The samples were sieved to $65 \mu \mathrm{m}$ and they were mixed with glass powder before filling the capillary. Rietveld refinements were performed with the help of Fullprof. ${ }^{16}$

Fig. 1 shows the $\mathrm{X}$ ray diffraction patterns and enlarged images of the peak with the highest intensity for representative sodium intercalated samples as well as for the host $\beta$ - $\mathrm{HfNCl}$. This peak corresponds to the 003 reflection and its position yields thus a direct measure of the interlayer separation of the intercalated and pristine compounds. The resolution achieved in our X-ray diffraction patterns allows the conclusion that all the sodium intercalated samples are a mixture of either the pristine $\beta-\mathrm{HfNCl}$ and a new intermediate phase or the intermediate phase and $\mathrm{Na}_{0.29} \mathrm{HfNCl}^{8}{ }^{8} \mathrm{We}$ did not obtain any biphasic sample containing only $\mathrm{Na}_{0.29} \mathrm{HfNCl}$ and the pristine. The average interlayer spacings observed for $\mathrm{Na}_{0.29} \mathrm{HfNCl}$ and $\beta-\mathrm{HfNCl}$ are 9.86(4) and 9.24(2) $\AA$ respectively whereas the intermediate phase shows a interlayer 

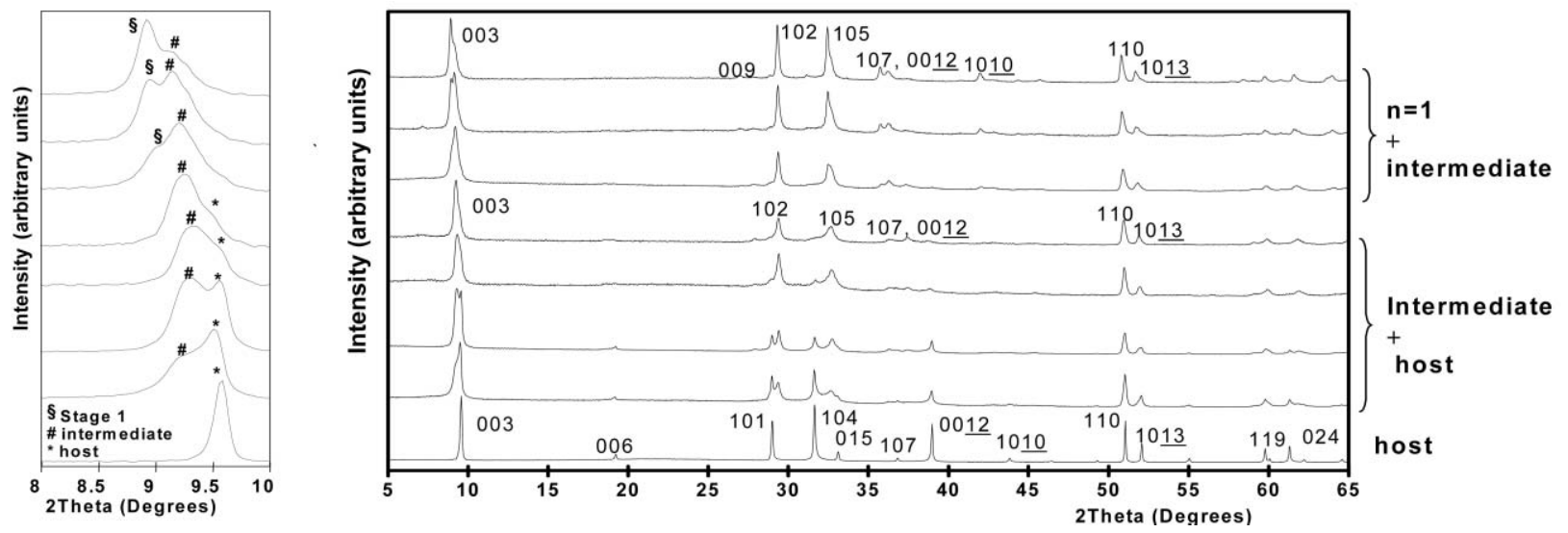

Fig. 1 X-ray diffraction patterns for $\mathrm{Na}_{x} \mathrm{HfNCl}$ samples obtained with different naphthyl-sodium: $\mathrm{HfNCl}$ ratios and enlarged images of the corresponding 003 reflections.

separation between 9.48 and $9.67 \AA$ depending on the sample. Stage determination is usually done by scanning the (001) reflections whose Bragg angles are related to the stage number (n) by the relation, $I_{n}=d_{\mathrm{s}}+(n-1) d_{\mathrm{s} 0}=1 /\left(2 \lambda \sin \theta_{1}\right) \AA$. In the present case $d_{\mathrm{s} 0}$ is the interlayer separation for the pristine, $c_{0} / 3=9.24 \AA, d_{\mathrm{s}}$ is the interlayer separation for the stage 1 phase and $\theta_{1}$ are the Bragg angles for the (001) reflections. Taking into account the previously published structural data, we should consider that the phase reported by Shamoto et al. ${ }^{8}$ $\mathrm{Na}_{0.29} \mathrm{HfNCl}$, corresponds to a diluted stage 1 intercalate of $\mathrm{HfNCl}$ with all of the van der Waals gaps filled with relative occupancy of $c a .50 \%$. For the $3 a$ sites occupied by the sodium atoms in this phase, $100 \%$ of occupancy would lead to 0.5 sodium atoms per formula. The observed spacings in our samples for the intermediate intercalate $\left(d_{\text {average }}=9.56(7) \AA\right)$ fit to the expression $d(n)=I_{n} / n=d_{\mathrm{s} 0}+\left(d_{\mathrm{s}}-d_{\mathrm{s} 0}\right) / n$ with $n=2, d_{\mathrm{s}}=9.86 \AA$ and $d_{\mathrm{s} 0}=9.24 \AA$ and consequently they are consistent with a second stage intercalate where one gallery out of two is filled with sodium atoms. From the above expression the calculated $d(2)$ is $9.55 \AA$. The dispersion of distances observed for the stage 2 phase is also observed for the stage 1 , which is indicative of the existence of dense and dilute intercalates. Dense and dilute stages as well as complex staging (i.e. stage superstructures with different intercalate densities in different layers) are frequently observed in intercalation compounds ${ }^{17,18}$ and may account for the observed spacings. The relative proportions observed for the pristine, stage 1 and the stage 2 phases in the different samples were found to depend on the concentration of the naphthyl-sodium solution as well as on the ratio $\mathrm{Na}: \mathrm{HfNCl}$. Both larger concentrations of the naphthylsodium solution and $\mathrm{Na}: \mathrm{HfNCl}$ ratios favoured the formation of the stage 1 with respect to the stage 2 . On the other hand, the experiments performed with a large excess of naphthyl sodium lead to disordered stage 1 phases and we observed the presence of impurities, typically sodium chloride that is indicative of the total reduction of the pristine to $\mathrm{HfN}$. The new stage 2 intercalate also showed broad reflections in some samples indicating low coherency along the $\mathrm{c}$ axis.

Fig. 2 shows the observed and calculated X-ray diffraction patterns for one of the samples containing the $n=1$ phase and the new intermediate intercalate. For the $n=1$ phase we used the coordinates of $\mathrm{Na}_{0.29} \mathrm{HfNCl}^{8}$ as initial atomic parameters. As

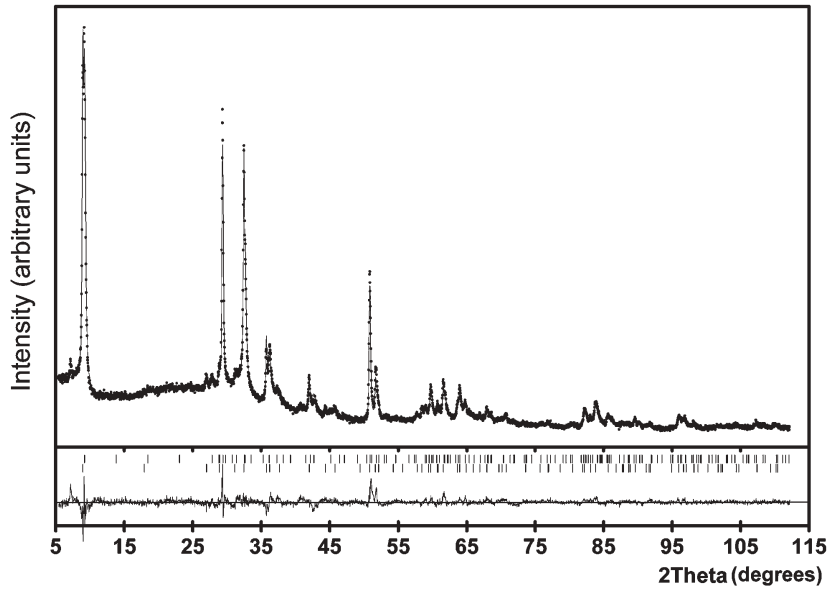

Fig. 2 Observed and calculated X-ray diffraction patterns for a sample containing the stage 1 and stage 2 phases of $\mathrm{Na}_{x} \mathrm{HfNCl}^{19}$

starting coordinates for the stage 2 phase we used both ordered and disordered structural models with YOF polytype and space group $R \overline{3} m$ that lead to similar agreement factors. Results in Fig. 2 correspond to an ordered superstructure with $a=3.58131(6)$, $c=57.752(6) \AA$, two crystallographically independent $\mathrm{HfNCl}$ units with occupancy factors of 1 and sodium atoms in the van der Waals gaps alternating along the $c$ axis to give the average composition $\mathrm{Na}_{0.125} \mathrm{HfNCl} .{ }^{19}$ The sodium stoichiometry was fixed to 0.125 during the refinement and in both cases soft constraints were imposed on $\mathrm{Hf}-\mathrm{Cl}, \mathrm{Hf}-\mathrm{N}$ and $\mathrm{N}-\mathrm{Cl}$ distances inside each $\mathrm{HfNCl}$ unit. However, the two $\mathrm{HfNCl}$ units were left to refine freely and independently. The refined structure showed different spacings between the double layers alternating along $c$, according with the filling by the sodium atoms of alternating van der Waals gaps in the stage 2 model.

Electrochemical intercalation experiments yield further evidence of the presence of intermediate phases between the pristine $\mathrm{HfNCl}$ and the $n=1$ intercalated phase. Indeed, the potential vs. composition profile (see Fig. 3) shows at least two shoulders, indicative of two-phase processes. ${ }^{20}$ The existence of these twophase regions is a clear proof of the appearance of intermediate intercalated phases. The fact that these are observed also in $\mathrm{ZrNCl}$ seems to indicate that this is a general behaviour within this family 


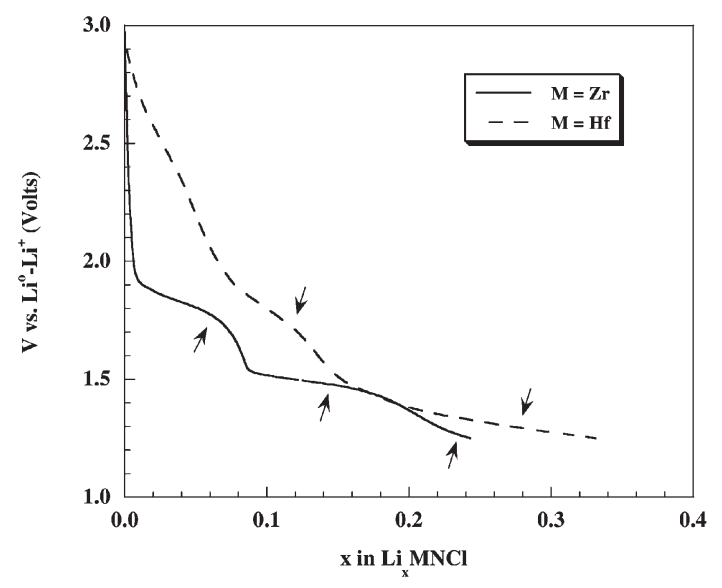

Fig. 3 Potential-composition profile for electrochemical lithium intercalation in $\mathrm{MNCl}(\mathrm{M}=\mathrm{Zr}, \mathrm{Hf})$ recorded in galvanostatic mode at $\mathrm{C} / 200$ between 3 and $1.2 \mathrm{~V}$ vs. $\mathrm{Li}^{0} / \mathrm{Li}^{+}$. Arrows indicate two-phase regions.

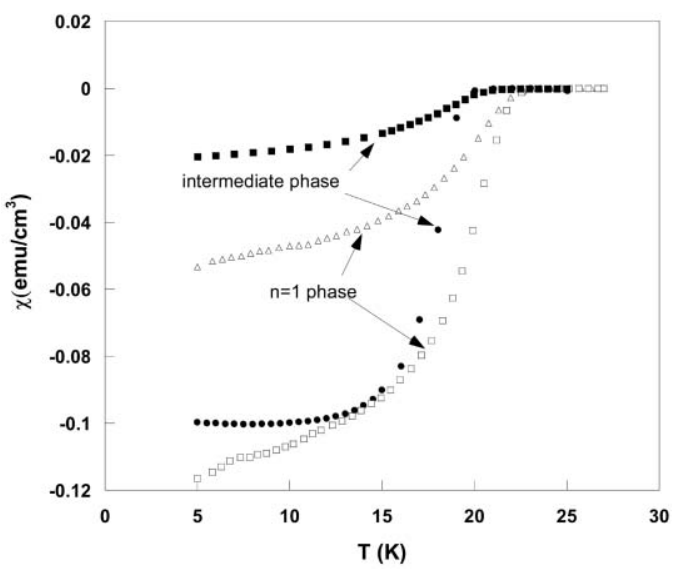

Fig. 4 Magnetic susceptibilities for different $\mathrm{Na}_{x} \mathrm{HfNCl}$ samples containing mixtures of the $n=1$ and the intermediate phase and the intermediate phase and the pristine.

of compounds, which is fully consistent with the existence of different intercalation stages. Unfortunately, the complete electrochemical study below $1 \mathrm{~V}$ vs. $\mathrm{Li}^{0} / \mathrm{Li}^{+}$was prevented by the interference of the reaction between lithium and the carbon used as a conducting additive in the fabrication of the electrodes. However, taking into account the $x$ scale, we can tentatively assign the process observed at $c a .1 .7 \mathrm{~V}$ in $\mathrm{HfNCl}$ to the formation of the stage 2 phase. With this assumption the shoulder at $2.4 \mathrm{~V}$ should correspond to a higher stage that we have not yet succeeded in isolating.

Fig. 4 shows the magnetic susceptibility measurements down to $4 \mathrm{~K}$ for four samples. The relative proportions of the different phases affects the observed critical temperature as well as the superconducting fraction. Samples containing the new stage 2 phase as the only sodium intercalated phase showed bulk superconductivity with a critical temperature of $20 \mathrm{~K}$ whereas the samples containing the stage 1 phase in different proportions showed a higher critical temperature, typically $23 \mathrm{~K}$. These results agree with the expected correlation between $T_{\mathrm{c}}$ and the doping level in this system and account for the different critical temperatures previously observed, ${ }^{2,8-13}$ but a complete elucidation of $T_{\mathrm{c}} v s . x$ dependencies along with the possible existence of higher stage phases certainly deserves further attention.

This work was supported by the Generalitat de Catalunya (grant 2001SGR 00361) and the Ministerio de Educación y Ciencia of Spain (grant MAT 2002-00439).

Judith Oró-Solé, ${ }^{a}$ Carlos Frontera, ${ }^{a}$ Benjamín Martínez, ${ }^{a}$ Daniel Beltrán-Porter, ${ }^{b}$ Maria Rosa Palacín ${ }^{a}$ and Amparo Fuertes*a ${ }^{a}$ Institut de Ciència de Materials de Barcelona (C.S.I.C.), Campus U.A.B., 08193, Bellaterra, Spain. E-mail: amparo.fuertes@icmab.es; Fax: 34 935805729; Tel: 34935801853

${ }^{b}$ Institut de Ciència de Materials de la Universitat de València, P.O. BOX 2085, Polígono "La Coma" s/n, 46980, Paterna, Spain

\section{Notes and references}

1 S. Yamanaka, K. Hohetama and H. Kawaji, Nature, 1998, 392, 580.

2 S. Yamanaka, H. Kawaji, K. Hotehama and M. Ohashi, Adv. Mater., 1996, 8, 771; H. Kawaji, K. Hotehama and S. Yamanaka, Chem. Mater., 1997, 9, 2127.

3 A. Fuertes, M. Vlassov, D. Beltrán-Porter, P. Alemany, E. Canadell, N. Casañ-Pastor and M. R. Palacín, Chem. Mater., 1999, 11, 203.

4 R. Juza and H. Z. Friedrichsen, Z. Anorg. Allg. Chem., 1964, 332, 173.

5 A. M. Fogg, J. S. O. Evans and D. O'Hare, Chem. Commun., 1998, 2269.

6 J. Oró-Solé, M. Vlassov, D. Beltrán-Porter, M. T. Caldés, V. Primo and A. Fuertes, Solid State Sci, 2002, 4, 475.

7 M. Fogg, V. M. Green and D. O'Hare, Chem. Mater., 1999, 11, 216.

8 S. Shamoto, K. Iizawa, M. Yamada, K. Ohoyama, Y. Yamaguchi and T. Kajitani, J. Phys. Chem. Solids, 1999, 60, 1431.

9 S. Shamoto, K. Izawa, Y. Asano, K. Ohoyama and T. Kajitani, Mol. Cryst. Liq. Cryst., 2000, 341, 515.

10 S. Yamanaka, K. Hotehama, T. Koiwasaki, H. Kawaji, H. Fukuoka, S. Shamoto and T. Kajitani, Phys. C, 2000, 341, 699.

11 A. Cross, A. Cantarero, D. Beltrán-Porter, J. Oró-Solé and A. Fuertes, Phys. Rev. B, 2003, 67, 104502.

12 A. M. Fogg, V. M. Green and D. O'Hare, J. Mater. Chem., 1999, 9, 1547.

13 M. Vlassov, M. R. Palacín, D. Beltrán-Porter, J. Oró-Solé, E. Canadell, P. Alemany and A. Fuertes, Inorg. Chem., 1999, 38, 4530.

14 M. S. Dresselhaus and G. Dresselhaus, Adv. Phys., 1981, 30, 139.

15 M. S. Dresselhaus, Intercalation in Layered Materials, Proceedings of the 10th Course of the Erice Summer School, Plenum, New York, 1986.

16 Fullprof 2000, See http://www-llb.cea.fr/fullweb/powder.htm. J. Rodríguez-Carvajal, Physica B, 1993, 192, 55.

17 K. K. Bardhan, G. Kirczenow, G. Jackle and J. C. Irwin, Phys. Rev. B, 1986, 33, 4149.

18 D. C. Dahn and R. R. Haering, Solid State Commun., 1982, 44, 29.

19 Structural data for the stage 2 phase: Chemical formula: $\mathrm{Na}_{0.125} \mathrm{HfNCl}$. Cell dimensions: $3.58131(6), c=57.752(6) \AA$. Space group: $R$ ??? $m$. $Z=12$. Number of experimental points: 3947 . Number of reflections: 159. Conventional $R$ factors corrected for background: $R_{\mathrm{p}}=19.6$, $R_{\mathrm{wp}}=17.8, R_{\exp }=11.23 . \chi^{2}=2.5 ; R_{\text {Bragg }}=4.13 \cdot{ }^{16}$ Refined atomic coordinates $(x / a, y / b, z / c)$ : $\operatorname{Hf}(1): 0,0,0.39677(6) ; \operatorname{Hf}(2): 0,0,0.10499(9)$; $\mathrm{N}(1): 0,0,0.06909(9) ; \mathrm{N}(2): 0,0,0.43592(6) ; \mathrm{Cl}(1): 0,0,0.19537(3) ; \mathrm{Cl}(2)$ : $0,0,0.30237(3)$; Na: 0,0,0. Soft constraints applied to the following bond distances: $\mathrm{Hf}(1)-\mathrm{Cl}(2) ; \mathrm{Hf}(1)-\mathrm{N}(1) ; \mathrm{N}(1)-\mathrm{Cl}(2) ; \mathrm{Hf}(2)-\mathrm{Cl}(1) ; \mathrm{Hf}(2)-\mathrm{N}(2)$ and $\mathrm{N}(2)-\mathrm{Cl}(1)$. Selected bond distances $(\AA)$ : $\operatorname{Hf}(1)-\mathrm{N}(1)(\times 3)$ : 2.0934(6); $\mathrm{Hf}(1)-\mathrm{N}(2): 2.261(6) ; \mathrm{Hf}(1)-\mathrm{Cl}(2)(\times 3): 2.792(3) ; \mathrm{Hf}(2)$ $\mathrm{N}(1): 2.073(2) ; \mathrm{Hf}(2)-\mathrm{N}(2)(\times 3): 2.0723(3) ; \mathrm{Hf}(2)-\mathrm{Cl}(1)(\times 3): 2.811(3)$; $\mathrm{Na}(1)-\mathrm{Cl}(2)(\times 6): 2.734(2)$. CCDC 268128 \& 268129. See http:// www.rsc.org/suppdata/cc/b5/b504289e/ for crystallographic data in CIF or other electronic format.

20 M. B. Armand, NATO Conference Proceeding Series VI. Mater. Sci. Vol 2. Ed. D. W. Murphy, J. Broadhead, B. C. H. Steele. 1980, Plenum Press, New York. p. 145-161. 